\title{
A NOTE ON LOWER RADICALS OF HEMIRINGS
}

\author{
Muhammad ZulfiQAR
}

\begin{abstract}
In this paper, we generalize a few results of [7, 10] for lower radical classes of rings, by using the limit ordinal construction for lower radical classes of hemirings.
\end{abstract}

\section{Introduction and preliminaries}

D. M. Olson and T. L. Jenksins [8] discussed general Radical Theory of Hemirings. The theory was further enriched by many authors (see $[4,12,13])$. The lower radicals were investigated by (see $[5,6,9,10,11])$ for radical classes of rings. Here we are interesting to generalize a several results of (see $[3,7$, 10]) in the frame work of hemiring which is quite different from ring theoretical approach discussed in (see $[3,7,10]$ ).

A semiring $(R,+, \cdot)$ is called a hemiring if

(i) ' + ' is commutative,

(ii) there exists an element $0 \in R$ such that 0 is the identity of $(R,+)$ and the zero element of $(R, \cdot)$, i.e., $0 r=r 0=0, \forall r \in R$.

If $I$ is an semi-ideals of $R$, then we denote $I \leq R$.

Lower radical classes for hemirings can be constructed similar to the construction of lower radicals for rings (see $[3,5,6,9,10,11]$ ).

First we include necessary preliminary, let $\omega$ be the universal class of all hemirings and $M$ be a sub-class of $\omega$ and let $M_{0}$ be the homomorphic closure of $M$ in $\omega$. For each $A \in \omega$, let $D_{1}(R)$ be the set of all semi-ideals of $R$. Inductively we define

$$
D_{n+1}(R)=\left\{I: I \text { is an semi-ideal of some hemiring in } D_{n}(R)\right\} .
$$

Let $D(R)=\bigcup_{n \in N} D_{n}(R), n=1,2,3, \ldots$ By using ring theoretical approach discussed in [11], we have

$$
£ M=\left\{R \in \omega: D(R / I) \cap M_{0} \neq 0 \text { for each proper semi-ideal } I \text { of } R\right\},
$$

Received March 20, 2008.

2000 Mathematics Subject Classification. 16N80.

Key words and phrases. hemiring, radical classes, universal class, semisimple class, homomorphically closed, $p$-semi-ideal, $k$-ideal, hereditary class, lower radical. 
is the Lee construction for lower radical determined by $M$, and $M \subseteq £ M$ (see also $[8,12,13])$.

Let $\omega$ be a universal class of (not necessarily associative) hemirings and let $A \subseteq \omega$. R. Wiegandt has given in [11] a construction for $£ A$, the lower radical class determined by $A$ in $\omega$. Using this construction, Leavitt and Hoffmann have proved in [3] that if $A$ is a hereditary class (if $K \in A$ and $I \leq K$, then $I \in A$ ), then $£ A$ is also hereditary. In this paper lower radical construction is given. As applications, a simple proof is given of the theorem of Leavitt and Hoffmann and a result of Y. L. Lee and R. E. Propes [7] for hemirings is extended to not necessarily associative hemirings.

Let $A \subseteq \omega$ be any class of hemirings. Define $R_{1}(A)$ to be the homomorphic closure of $A$. Proceeding inductively, let $\mu$ be an ordinal exceeding one and suppose the classes $R_{\lambda}(A)$ have been defined for all $\lambda<\mu$. If $\mu$ is not a limit ordinal, define

$$
R_{\mu}(A)=\left\{K \in \omega: I, K / I \in R_{\mu-1}(A) \text { for some } I<K\right\} .
$$

If $\mu$ is a limit ordinal, define $R_{\mu}(A)=\left\{K \in \omega: K\right.$ contains a chain $I_{\gamma}$ of semi-ideals such that each $I_{\gamma} \in \bigcup_{\lambda<\mu} R_{\lambda}(A)$, and $\left.K=\cup I_{\gamma}\right\}$. Finally define $R(A)=\cup R_{\lambda}(A)$, where the union is taken over all ordinals $\lambda$. For undefined terms of hemirings we may refer (see $[1,2,8]$ ).

\section{Lower radicals}

We extend the result of [7] by using the above limit ordinal construction of lower radical for hemiring which is indeed provides an excellent and different approach to handle the many results of [7] in the frame work of hemiring.

The following theorem was proved by F. A. Szasz [10] for rings. Here we generalize it for hemiring, which can be obtained on the line of rings theoretical approach.

Theorem 2.1. Let $\omega$ be a universal class of hemiring and let $A \subseteq \omega$. Then $A$ is a radical class in $\omega$ if and only if the following conditions are satisfied:

(1) A is homomorphically closed,

(2) If $I, K / I \in A$, then $K \in A$,

(3) The union of a chain of $A$-semi-ideals of a $\omega$-hemiring $K$ is again an A-semi-ideal of $K$.

The following theorem is obvious.

Theorem 2.2. If $\lambda$ and $\mu$ are ordinals with $\lambda \leq \mu$, then $R_{\lambda}(A) \subseteq R_{\mu}(A)$.

Theorem 2.3. For every ordinal $\lambda \geq 1, R_{\lambda}(A)$ is homomorphically closed. Hence $R(A)$ is homomorphically closed.

Proof. $R_{1}(A)$ is homomorphically closed. Let $\mu>1$ be an ordinal, and suppose $R_{\lambda}(A)$ is homomorphically closed for all $\lambda<\mu$. Let $K \in R_{\mu}(A)$ and let $I<K$. If $\mu$ is a limit ordinal, there is a chain $\left\{I_{\gamma}\right\}$ of semi-ideals of $K$ such that 
$I_{\gamma} \in R_{\lambda}(A)$ with $\lambda<\mu$ and such that $K=\cup I_{\gamma}$. But $\left\{\left(I+I_{\gamma}\right) / I\right\}$ is a chain of semi-ideals of $K / I$, and $K / I$ is its union. Since $\left(I+I_{\gamma}\right) / I \cong I_{\gamma} /\left(I^{*} \cap I_{\gamma}\right)$, where $I^{*}$ is a $k$-ideal generated by $I$ (see $\left.\left.[8,13]\right)\right)$ each of these semi-ideals is a homomorphism of some $I_{\gamma}$, and thus by induction hypothesis each $\left(I+I_{\gamma}\right) / I \in$ $R_{\lambda}(A)$ with $\lambda<\mu$. This implies that $K / I \in R_{\mu}(A)$.

Now suppose $\mu-1$ exists. Then $K$ contains an semi-ideal $J$ so that $J, K / J \in$ $R_{\mu-1}(A)$. By the induction hypothesis, $(J+I) / I \in R_{\mu-1}(A)$ and $K /(I+J) \in$ $R_{\mu-1}(A)$, since the former is a homomorphic image of $J$ and latter of $K / J$. Since $[R / I] /\left[(J+I)^{*} / I\right] \cong R /(J+I)$, where $(J+I)^{*}$ is a $k$-ideal generated by $(J+I)$ (see $[12]), K / I \in R_{\mu}(A)$. Thus by transfinite induction $R_{\mu}(A)$ is homomorphically closed for ordinal $\mu$. It follows immediately that $R(A)$ is homomorphically closed.

We now show that $R(A)$ satisfies Conditions 2 and 3 of Theorem 2.1.

Theorem 2.4. Let $K \in \omega$, and let $\left\{I_{\lambda}\right\}$ be a chain of $R(A)$-semi-ideals of $K$. Then $\cup I_{\lambda}$ is an $R(A)$-semi-ideal of $K$.

Proof. Since $K$ is a set, there is by Theorem 2.2 an ordinal $\mu$ with the property that $I_{\lambda} \in R_{\mu}(A)$ for each $\lambda$. Let $\delta$ be a limit ordinal exceeding $\mu$, then $\cup I_{\lambda} \in$ $R_{\delta}(A)$.

Theorem 2.5. Let $K \in \omega$, and suppose $K$ contains an semi-ideal $I \in R(A)$ such that $K / I \in R(A)$. Then $K \in R(A)$.

Proof. By Theorem 2.2, there is an ordinal $\mu$ such that $I, K / I \in R_{\mu}(A)$. This implies that $K \in R_{\mu+1}(A)$.

Theorem 2.6. $R(A)=£(A)$.

Proof. By Theorem 2.1 and Theorems 2.3, 2.4, and 2.5, $R(A)$ is a radical class in $\omega$. By the minimality of $£(A)$ among radical classes in $\omega$ which contain $A$, it is enough to show $R(A) \subseteq £(A)$. This is accomplished by proving $R_{\lambda}(A) \subseteq £(A)$ for every ordinal $\lambda$. Clearly $R_{1}(A) \subseteq £(\mathrm{~A})$. Let $\mu$ be an ordinal exceeding one, and assume $R_{\lambda}(A) \subseteq £(A)$ for all ordinals $\lambda<\mu$. Let $K \in R_{\mu}(A)$. If $\mu$ is a limit ordinal, $K$ be the union of a chain of semi-ideals from the classes $R_{\lambda}(A)$, where $\lambda<\mu$. Thus by induction hypothesis $K$ is the union of $£(A)$-semi-ideals, so $K \in £(A)$ by Theorem 2.1. If $\mu$ is not a limit ordinal, there is an semi-ideal $I$ of $K$ such that $I \in R_{\mu-1}(A) \subseteq £(A)$ and $K / I \in R_{\mu-1}(A) \subseteq £(A)$. Again, $K \in £(A)$ by Theorem 2.1. Thus $R_{\mu}(A) \subseteq £(A)$ for all ordinals $\mu \geq 1$.

We now give a simple proof of the following theorem which appears in [3]. Other results of the form $A$ has property $\rho$ implies $£ A$ has property may, perhaps, be provable in a similar way. The following theorem was proved by A. E. Hoffman and W. G. Leavitt [3] and we generalize it in the frame work of hemirings. Here we give a proof of this theorem which is entirely different from [3]. 
Theorem 2.7. Let $A \subseteq \omega$ where $\omega$ is some universal class of hemiring. Then if $A$ is hereditary, so is $£(A)$.

Proof. We prove that $R_{\mu}(A)$ is hereditary for each $\mu \geq 1$. This is easily seen to be true if $\mu=1$. Thus, assume $\mu>1$, and suppose $R_{\lambda}(A)$ is a hereditary class for each $\lambda<\mu$. Let $K \in R_{\mu}(A)$, and suppose $I \leq K$. If $\mu$ is a limit ordinal, $K=\cup I_{\gamma}$ where $\left\{I_{\gamma}\right\}$ is a chain of semi-ideals each belonging to one of the (hereditary) classes $R_{\lambda}(A), \lambda<\mu$. But then $I=\cup\left(I_{\gamma} \cap I\right)$ so $I \in R_{\mu}(A)$. If $\mu$ is not a limit ordinal, there is an semi-ideal $J$ of $K$ so that $J, K / J \in R_{\mu-1}(A)$. Since $R_{\mu-1}(A)$ is hereditary, $I \cap J \in R_{\mu-1}(A)$ and $(J+I) / J \cong I /\left(I \cap J^{*}\right) \in$ $R_{\mu-1}(A)$, where $J^{*}$ is a $k$-ideal generated by $J$ (see $\left.\left.[8,13]\right)\right)$. This implies that $I \in R_{\mu}(A)$.

If $\rho$ is a radical class of hemiring then its semisimple class is denoted by $\mathrm{S} \rho$. The proof of Theorem 2.9 requires the following lemma.

Lemma 2.8. If $\rho$ is a radical class in $\omega$ and for some $K \in \in \omega$ a subhemiring $K \subseteq K^{\prime}$ is the set theoretic union of $\rho$-semi-ideals of $\bar{K}$, then $K \in \rho$.

Proof. If $K=\cup I_{\lambda}$ not belong to $\rho$, then $K / I \in S \rho=\{T \in \omega: T$ has no nonzero $\rho$-semi-ideals $\}$ for some $I \neq K$. By $\lambda$, we have $I_{\lambda} \nsubseteq \subseteq I$, so

$$
\left(I_{\lambda}+I\right) / I \cong I_{\lambda} /\left(I^{*} \cap I_{\lambda}\right)
$$

where $I^{*}$ is a $k$-ideal generated by $I$ (see $\left.[8,13]\right)$ ) is a nonzero $\rho$-semi-ideal of $K / I$. This contradiction proves that $K \in \rho$.

The following theorem was proved by Y. L. Lee and R. E. Propes [7] and we generalize it in the frame work of hemirings. Here we give a proof of this theorem which is entirely different from [7].

Theorem 2.9. If $A_{1}$ and $A_{2}$ are homomorphically closed, hereditary classes of $\omega$-hemirings, then $£\left(A_{1} \cap A_{2}\right)=£ A_{1} \cap £ A_{2}$.

Proof. Trivially $£\left(A_{1} \cap A_{2}\right) \subseteq £ A_{1} \cap £ A_{2}$. Since $K \in £ A_{1} \cap £ A_{2}$ if and only if $K \in R_{\gamma}\left(A_{1}\right) \cap R_{\gamma}\left(A_{2}\right)$ for some ordinal number $\gamma$. It suffices to prove $R_{\gamma}\left(A_{1}\right) \cap R_{\gamma}\left(A_{2}\right) \subseteq £ A_{1} \cap £ A_{2}$ for each ordinal $\gamma \geq 1$. This is clear for $\gamma=1$. Let $\mu$ be an ordinal number greater 1 and suppose $R_{\lambda}\left(A_{1}\right) \cap R_{\lambda}\left(A_{2}\right) \subseteq$ $£\left(A_{1} \cap A_{2}\right)$ for each ordinal $\lambda<\mu$. Let $K \in R_{\mu}\left(A_{1}\right) \cap R_{\mu}\left(A_{2}\right)$. If $\mu$ is a limit ordinal, $K$ is the union of a chain $\left\{I_{\gamma}\right\}, \gamma \in C$ of semi-ideals each belonging to one of the classes $R_{\lambda}\left(A_{1}\right)$ for $\lambda<\mu$. Also $K$ is the union of a chain $\left\{J_{\delta}\right\}$, $\delta \in D$ of semi-ideals each belonging to one of the classes $R_{\lambda}\left(A_{2}\right)$ for $\lambda<\mu$. If $x \in K, x \in J_{\delta}$ for some $\delta \in D$ and $x \in I_{\gamma}$ for some $\gamma \in C$, so $x \in J_{\delta} \cap I_{\gamma}$ for some $(\delta, \gamma) \in D \times C$. Since $J_{\delta} \in R_{\lambda}\left(A_{2}\right)$ for $\lambda<\mu$, and since $R_{\lambda}\left(A_{2}\right)$ is hereditary (see proof of Theorem 2.7), $J_{\delta} \cap I_{\alpha} \in R_{\lambda}\left(A_{2}\right)$. Similarly $J_{\delta} \cap I_{\gamma} \in$ $R_{\eta}\left(A_{1}\right)$ for some $\eta<\mu$. Thus $J_{\delta} \cap I_{\gamma} \in R_{\beta}\left(A_{1}\right) \cap R_{\beta}\left(A_{2}\right)$, where $\beta=\max [\eta, \lambda]$. Since $\beta<\mu$, the induction hypothesis so that $K$ is the set-theoretic union of $£\left(A_{1} \cap A_{2}\right)$-semi-ideals. So, by Lemma $2.8, K \in £\left(A_{1} \cap A_{2}\right)$. Now suppose $\mu-1$ exists, and let $K \in R_{\mu}\left(A_{1}\right) \cap R_{\mu}\left(A_{2}\right)$. Then there exist semi-ideals $I$ and 
$J$ such that $I, K / I \in R_{\mu-1}\left(A_{1}\right)$ and $J, K / J R_{\mu-1}\left(A_{2}\right)$. Since $R_{\mu-1}\left(A_{1}\right)$ and $R_{\mu-1}\left(A_{2}\right)$ are hereditary, $I \cap J \in R_{\mu-1}\left(A_{1}\right) \cap R_{\mu-1}\left(A_{2}\right)$ so $I \cap J \in £\left(A_{1} \cap A_{2}\right)$. Since $R_{\mu-1}\left(A_{1}\right)$ is homomorphically closed (Theorem 2.3),

$$
I /\left(I \cap J^{*}\right) \cong(I+J) / J \in R_{\mu-1}\left(A_{1}\right),
$$

where $J^{*}$ is a $k$-ideal generated by $J$ (see $[8,13]$ ). Since $R_{\mu-1}\left(A_{2}\right)$ is hereditary, $(I+J) / J$, as an semi-ideal of $K / J$ is a member of $R_{\mu-1}\left(A_{2}\right)$. Thus

$$
I /\left(I \cap J^{*}\right) \cong(I+J) / J \in R_{\mu-1}\left(A_{1}\right) \cap R_{\mu-1}\left(A_{2}\right) \subseteq £\left(A_{1} \cap A_{2}\right),
$$

where $J^{*}$ is a $k$-ideal generated by $J$ (see $[8,13]$ ). Thus $I \cap J \in R_{\mu-1}\left(A_{1}\right) \cap$ $R_{\mu-1}\left(A_{2}\right) \subseteq £\left(A_{1} \cap A_{2}\right)$ and $I /(I+J) \in R_{\mu-1}\left(A_{1}\right) \cap R_{\mu-1}\left(A_{2}\right) \subseteq £\left(A_{1} \cap A_{2}\right)$.

Thus since $I \cap J \in £\left(A_{1} \cap A_{2}\right)$ and $I /(I+J) \in £\left(A_{1} \cap A_{2}\right), I \in £\left(A_{1} \cap A_{2}\right)$. Similarly, $J \in £\left(A_{1} \cap A_{2}\right)$ so that $I+J$ is an $£\left(A_{1} \cap A_{2}\right)$-semi-ideal of $K$. Also $K /(I+J) \in R_{\mu-1}\left(A_{1}\right) \cap R_{\mu-1}\left(A_{2}\right) \subseteq £\left(A_{1} \cap A_{2}\right)$ since it is homomorphically image of both $K / J$ and $K / I$. Thus, since $I+J \in £\left(A_{1} \cap A_{2}\right)$ and $K /(I+J) \in$ $£\left(A_{1} \cap A_{2}\right)$, we have that $K \in £\left(A_{1} \cap A_{2}\right)$. We have shown that $R_{\mu}\left(A_{1}\right) \cap$ $R_{\mu}\left(A_{2}\right) \subseteq £\left(A_{1} \cap A_{2}\right)$ which proves the theorem.

\section{References}

[1] J. S. Golan, The theory of semirings with applications in mathematics and theoretical computer science, Pitman Monographs and Surveys in Pure and Applied Mathematics, 54. Longman Scientific \& Technical, Harlow; copublished in the United States with John Wiley \& Sons, Inc., New York, 1992.

[2] U. Hebisch and H. J. Weinert, Semirings: algebraic theory and applications in computer science, Translated from the 1993 German original. Series in Algebra, 5. World Scientific Publishing Co., Inc., River Edge, NJ, 1998.

[3] A. E. Hoffman and W. G. Leavitt, Properties inherited by the lower radical, Portugal. Math. 27 (1968), 63-66.

[4] D. R. LaTorre, On h-ideals and k-ideals in hemirings, Publ. Math. Debrecen 12 (1965), 219-226.

[5] Y. L. Lee, On the construction of upper radical properties, Proc. Amer. Math. Soc. 19 (1968), 1165-1166.

[6] 393-395.

[7] Y. L. Lee and R. E. Propes, On intersections and unions of radical classes, J. Austral. Math. Soc. 13 (1972), 354-356.

[8] D. M. Olson and T. L. Jenksins, Radical theory for hemirings, J. Natur. Sci. Math. 23 (1983), no. 1, 23-32.

[9] H. J. le Roux and G. A. P. Heyman, A note on the lower radical, Publ. Math. Debrecen 28 (1981), no. 1-2, 11-13.

[10] F. A. Szász, Radicals of Rings, Mathematical Institute Hungarian Academy of Sciences, 1981.

[11] R. Wiegandt, Radical and Semisimple Classes of Rings, Queen's Papers in Pure and Applied Mathematics, No. 37. Queen's University, Kingston, Ont., 1974.

[12] S. M. Yusuf and M. Shabir, Radical classes and semisimple classes for hemirings, Studia Sci. Math. Hungar. 23 (1988), no. 1-2, 231-235.

[13] M. Zulfiqar, The sum of two radical classes of hemirings, Kyungpook Math. J. 43 (2003), no. 3, 371-374. 
Department of Mathematics

Govt. College University Lahore

LAHORE 54000, PAKISTAN

E-mail address: mzulfiqarshafi@hotmail.com 